\title{
The superficial palmar branch of the radial artery: a corrosion cast study
}

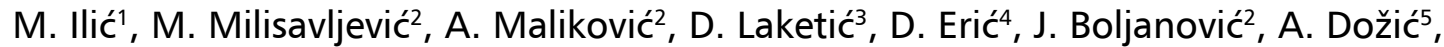 \\ B.V. Štimec ${ }^{6}$ R. Manojlović \\ 'Institute for Orthopaedic Surgery and Traumatology, Clinical Centre of Serbia, Faculty of Medicine, \\ University of Belgrade, Serbia \\ 2Laboratory for Vascular Anatomy, Institute of Anatomy, Faculty of Medicine, University of Belgrade, Serbia \\ ${ }^{3}$ Medical Department, Faculty of Sport and Physical Education, University of K. Mitrovica, Serbia \\ ${ }^{4}$ Department of Plastic, Reconstructive, and Hand Surgery, Faculty of Medicine, University of East Sarajevo, Foča, \\ Republic of Srpska, Bosnia and Herzegovina \\ 5Institute of Anatomy, Faculty of Dentistry, University of Belgrade, Serbia \\ ${ }^{6}$ Faculty of Medicine, Department of Cellular Physiology and Metabolism, Anatomy Sector, University of Geneva, \\ Switzerland
}

[Received: 10 January 2018; Accepted: 14 March 2018]

Background: Surgical procedures such as thenar flaps and radial artery (RA) harvesting call for an elaborate anatomical study of the RA's superficial palmar branch (SPB). The aim of this study was to describe the branching pattern of this vessel related to the morphometric characteristics and variations of this artery.

Materials and methods: Twenty 4\% formalin solution-injected hands were dissected. For the morphometric study we used another group of 35 human hands of adult persons, injected with methyl methacrylate fluid into the ulnar and radial arteries. As soon as polymerisation was completed, a $40 \%$ solution of potassium hydroxide was applied for corrosion. The vascular arterial casts were examined under the stereoscopic microscope and precise drawings of each specimen were made.

Results: In the majority of cases (75\%) SPB passed superficially, over the abductor pollicis brevis muscle. The mean diameter of the SPB, very variable depending on its length and field of supply, was $1.52 \pm 0.49 \mathrm{~mm}$, ranging from 0.8 to $2.7 \mathrm{~mm}$. Developed SPB type, was present in $31.4 \%$ of hands, with the diameter of $1.7 \mathrm{~mm}$ and larger (mean $1.95 \mathrm{~mm}$ ), continuing distally to become the radialis indicis artery, with an average calibre of $1.2 \mathrm{~mm}$, and with important branches to the thumb. In most hands (68.6\%), the hypoplastic SPB, was present, with a mean diameter of $1.17 \mathrm{~mm}$, and the field of supply within the thenar area.

Conclusions: Knowledge of the SPB dominance and existence of anastomotic vessels in its field of supply are of importance to avoid the risk of possible ischaemic sequelae in the hand associated with harvesting the RA. (Folia Morphol 2018; 77, 4: 649-655)

Key words: superficial palmar branch, radial artery, anastomoses, radialis indicis artery 


\section{INTRODUCTION}

Classical anatomical textbooks describe the superficial palmar branch (SPB) as the last side branch of the radial artery (RA) before it enters the anatomical snuff-box and makes the curve around the carpus. The SPB goes through or, occasionally, over the thenar muscles, sometimes anastomosing with the termination of the ulnar artery (UA), completing the superficial palmar arch (SPA) $[16,20]$.

Anatomical studies have seldom dealt with the role of SPB in the cutaneous blood supply of the thenar eminence itself $[4,17,21,26]$, or the arterial supply of the thumb $[2,15,26]$, and only rare data is available on the calibre of the SPB $[2,5,17]$. To our knowledge, there are no articles in the literature describing the branching pattern related to the morphometric features and variations of the SPB.

The position and the variations of the SPB, as well as the precise vascular pattern, related to its diameter and anastomoses, are important issues in plastic and reconstructive hand surgery. The thenar flaps are considered as useful techniques for reconstruction in numerous cases of palmar skin defects or loss of fingertip soft tissue with tendon and bone [7, 17, 22]. The RA harvesting for coronary revascularisation requires the evaluation of its collateral circulation to avoid hand ischaemia [1]. Therefore, knowledge on relevant anatomic data and potential surgical significance of area supplied by the SPB provides scientific and practical importance for this anatomical study $[8,18,24]$.

The aim of this detailed study was, firstly, to present all the possible anatomical variations of the SPB of RA, and secondly, to provide important anatomical basis for improving the safety of new flap designs and microsurgical techniques. Moreover, we underlined the surgical significance of the SPB dominance type based on the obtained anatomical data.

\section{MATERIALS AND METHODS}

Twenty formalin injected hands (8 female and 12 male) from the collection of the Laboratory for Vascular Anatomy, were dissected and the positions of SPBs of RAs, related to the thenar surface, were analysed for this study. For the morphometric comparisons we used another group of 35 human hands (16 right, 19 left) of adults (13 female and 22 male), with a mean age of 51.4 (range 26-65) years, with the upper limb vasculature completely preserved. For a better understanding and accurate presentation of relationships between the branches we prepared vascular arterial casts of the hands. A mixture of monomer and polymer partitions of methyl methacrylate was injected under manual pressure into the ulnar and radial arteries simultaneously. As soon as polymerisation and hardening of plastic was completed, an immersion in $40 \%$ solution of potassium hydroxide was applied for corrosion, and after 7 days of soft tissues digestion the whole cast was rinsed in a hot water. The hand blood vessels were examined under the stereoscopic microscope Leica MZ6), precise drawings of each specimen were made, measurements of the vessels were accomplished under ocular micrometre, and digital photos taken. The main advantage of corrosion cast technique is that it enables a precise three-dimensional spatial distinction between the vessels, a clear definition of anastomoses, and finally, this method allows for each vessel to be followed up to the level of its smallest rami, thus permitting a detailed description of its irrigation territory, much better than any dissection. A drawback of this method is the length of time required to complete the preparation and study in detail of even one single specimen. The study protocol was approved by the Ethics Committee of the Faculty of Medicine, University of Belgrade, Belgrade, Serbia (No. 29/VI-1; Date 19-6-2013). The obtained set of specimens served as a useful demonstration tool in teaching medical students, as an adjunct in the collection of the Museum of Human Anatomy in Institute of Anatomy, Faculty of Medicine of University of Belgrade, as well as in preparation of the Atlas of the Human Body [25].

\section{RESULTS}

In all the studied hands (100\%), the distal portion of the RA, lateral to the tendon of flexor carpi radialis muscle and at the level of the wrist joint and the styloid process of the radius, gave off always one artery - the SPB (Figs. 1-3). The mean diameter of the RA at the level of SPB origin was $2.9 \pm 0.34$ (range 2.4-3.5) $\mathrm{mm}$. The SPB diameter, measured at its emerging from the $\mathrm{RA}$, ranged from 0.8 to 2.7 (mean $1.52 \pm 0.49$ ) $\mathrm{mm}$, i.e. it was very variable depending on its length and irrigation area (Table 1). Regardless of the size of RA's SPB, in the majority of cases (15 out of 20 dissected hands - 75\%), it passed superficially through the subcutaneous tissue of the thenar eminence, over the surface of the abductor pollicis brevis muscle (Fig. 1A). Less frequently ( $25 \%$ of hands) the artery was deeply positioned, following the intermuscular space between the above mentioned muscle and the opponens pollicis muscle underneath (Fig. 1B). 


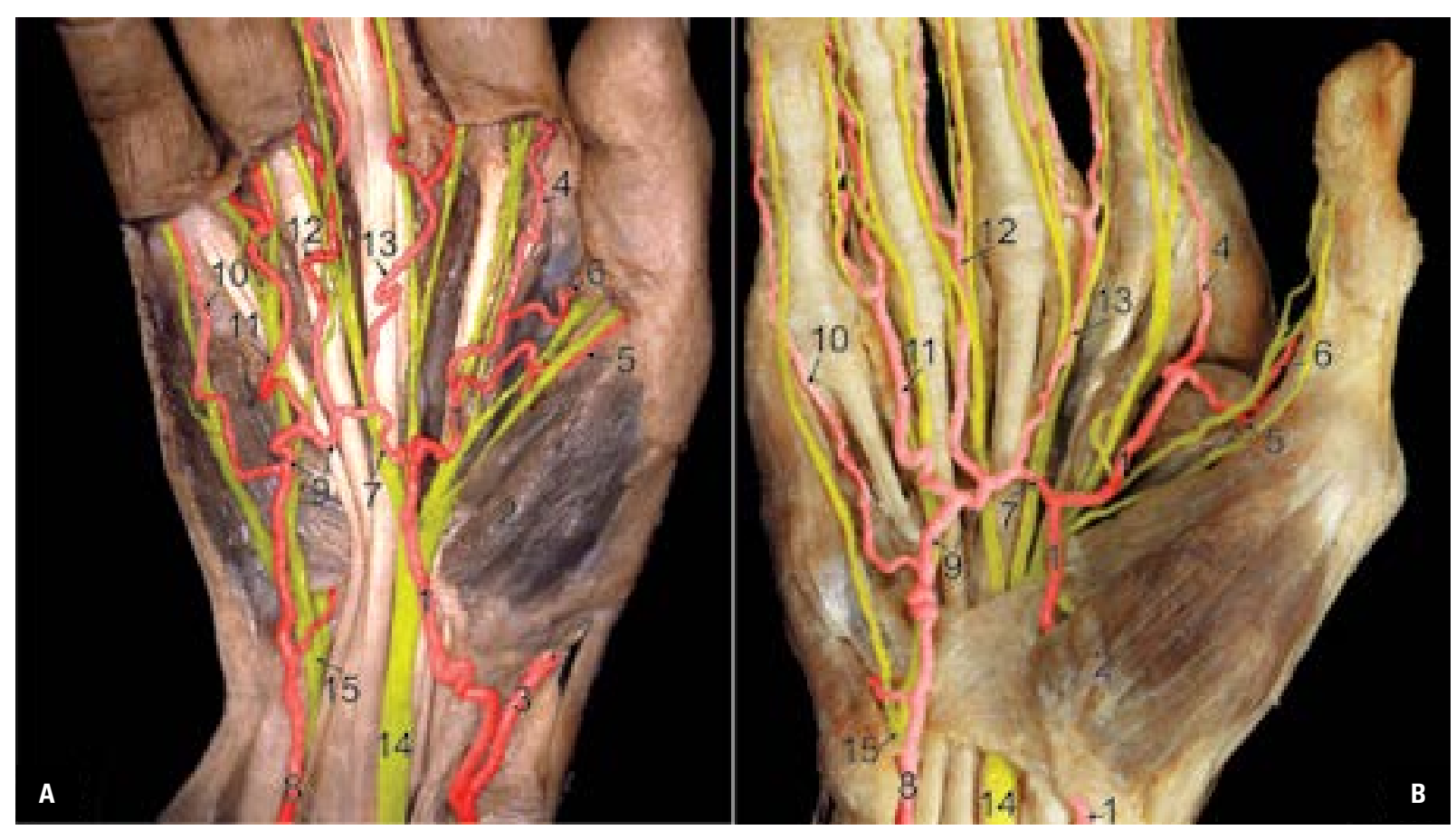

Figure 1. Dissections of the right hands, with arteries additionally coloured in red and nerves in yellow; palmar view: A. Well-developed superficial palmar branch (SPB) (1) with superficial position over the abductor pollicis brevis muscle (2); B. Well-developed SPB (1) passing deep to the abductor pollicis brevis muscle (2); 3 — radial artery; 4 — radialis indicis artery; 5 — radialis pollicis palmar artery; 6 — ulnaris pollicis palmar artery; 7 - communicating branch; 8 - ulnar artery; 9 - superficial palmar arch; 10 - ulnaris digiti minimi palmar artery; 11 - third common palmar digital artery III; 12 - second common palmar digital artery II; 13 - first common palmar digital artery I; 14 — median nerve; 15 — ulnar nerve.

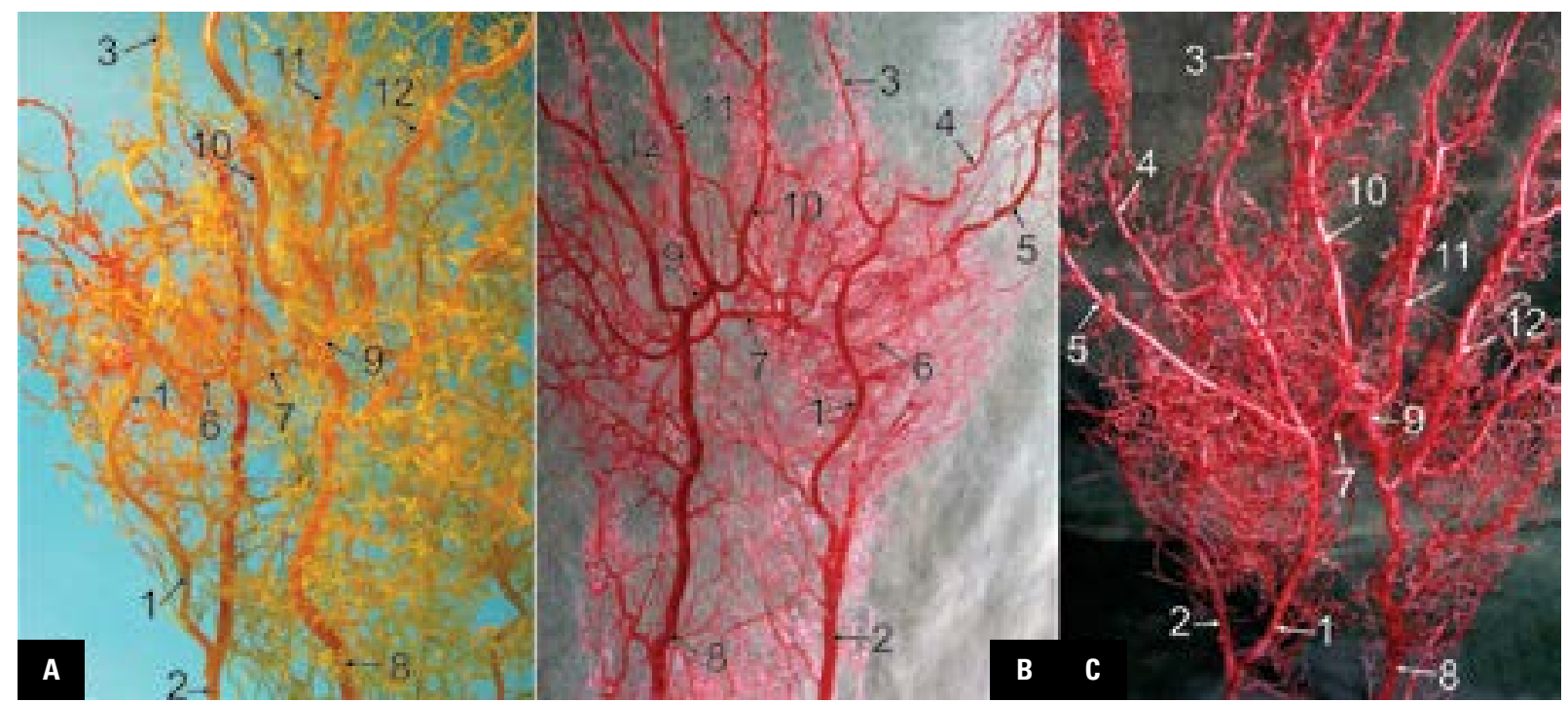

Figure 2. Corrosion casts of three hands with prominent superficial palmar branch (SPB) (1) of the radial artery (2), palmar view, continuing as: A. Left radialis indicis artery (RIA) (3); B. Right RIA (3) and ulnaris pollicis palmar artery (UPPA) (4); C. Left RIA (3), UPPA (4) and radialis pollicis palmar artery (5); 6 - princeps pollicis artery; 7 - deep palmar arch; 8 - ulnar artery; 9 - superficial palmar arch; 10 - common palmar digital artery l; 11 — common palmar digital artery II; 12 — common palmar digital artery III.

In view of the morphometric and topographic features of the SPB we determined two main types: well-developed and hypoplastic artery.

Type A. Well-developed SPB type, found in 11 (31.4\%) hands, was characterised by one prominent arterial stem of SPB, with the diameter of $1.7 \mathrm{~mm}$ and above, ranging from 1.7 to 2.7 (mean 1.95) $\mathrm{mm}$, continuing from the thenar area into the metacarpal part of the hand to become finally the radialis indicis artery (RIA), along the lateral side of the index finger (Figs. 1, 2). 


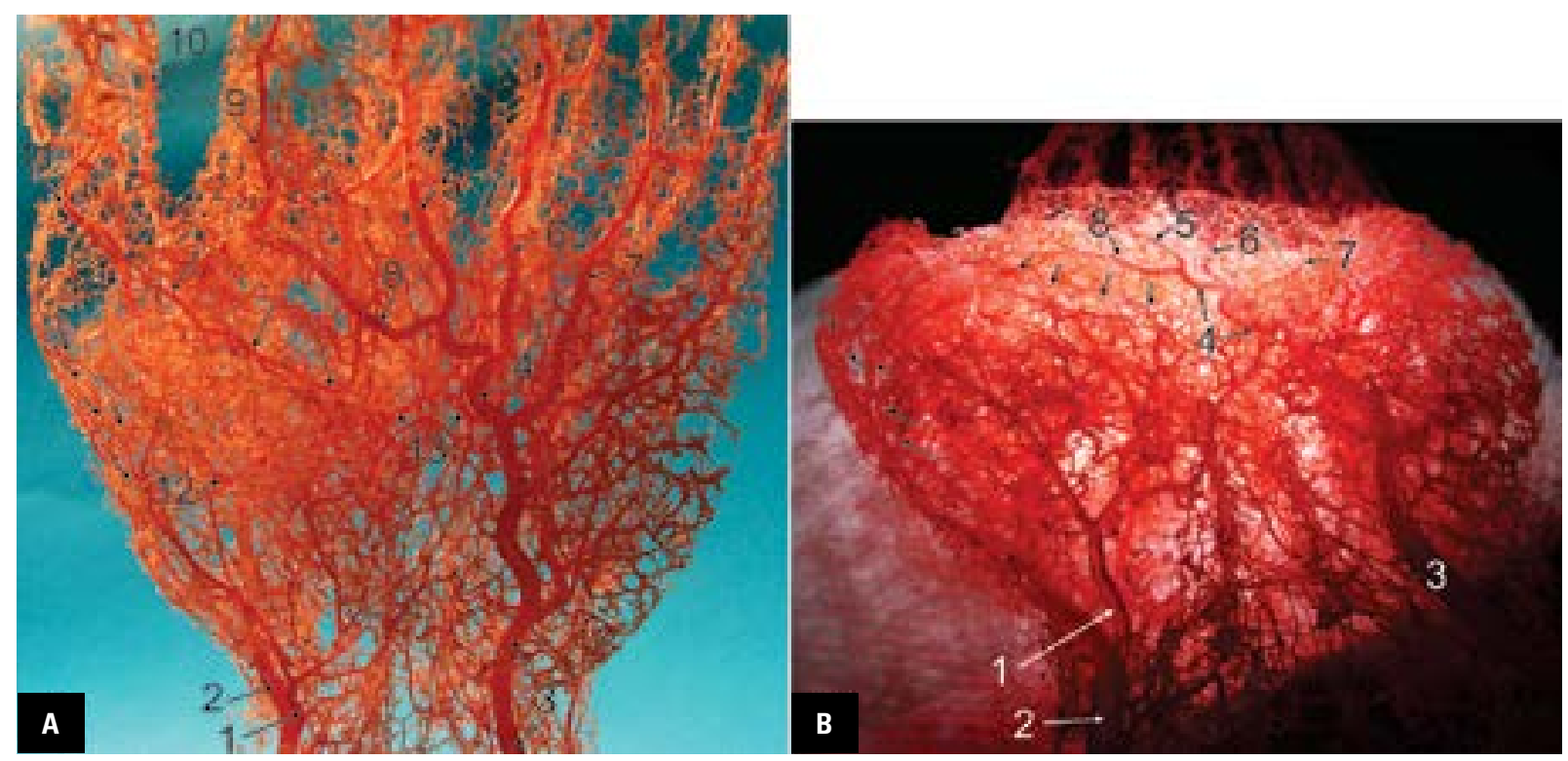

Figure 3. The vascular corrosion cast of the left hand showing the vascular territory and anastomotic subcutaneous vessels (arrows) of the hypoplastic superficial palmar branch (1) of radial artery (2): A. Palmar view; B. Palmar view from the level of a root of hand distally onward; 3 - ulnar artery; 4 - superficial palmar arch; 5 - common palmar digital artery I; 6 - common palmar digital artery II; 7 - common palmar digital artery III; 8 - communicating branch; 9 — radialis indicis artery; 10 - ulnaris pollicis palmar artery; 11 - radialis pollicis palmar artery; 12 - princeps pollicis artery; 13 - deep palmar arch.

Table 1. Morphometric characteristics of the radial artery, superficial palmar branch (SPB) and its braches

\begin{tabular}{lcc}
\hline Artery & $\begin{array}{c}\text { Incidence } \\
\mathbf{N}(\%)\end{array}$ & $\begin{array}{c}\text { Diameter [mm] } \\
\text { Range (mean } \pm \text { SD) }\end{array}$ \\
\hline Radial artery & $35(100 \%)$ & $2.4-3.5(2.9 \pm 0.34)$ \\
SPB & $35(100 \%)$ & $0.8-2.7(1.52 \pm 0.49)$ \\
Well-developed SPB & $11(31.4 \%)$ & $1.7-2.7(1.95)$ \\
$\quad$ RIA & $11(31.4 \%)$ & $1.0-1.5(1.2)$ \\
UPPA & $6(17.1 \%)$ & $1.0-1.3(1.15)$ \\
$\quad$ UPPA + RPPA & $4(11.4 \%)$ & {$[1.0-1.4(1.3)]+[1.0-1.35(1.2)]$} \\
Hypoplastic SPB & $24(68.6 \%)$ & $0.8-1.5(1.17)$ \\
\hline
\end{tabular}

RIA — radialis indicis artery; UPPA — ulnaris pollicis palmar artery; RPPA — radialis pollicis palmar artery; SD — standard deviation

Calibre of the RIA was on the average 1.2 (range 1.0$-1.5) \mathrm{mm}$ measured at the level of the index finger's root (Table 1). A long and well-developed SPB was the dominant source of the blood supply to the thenar skin and muscles, and to the lateral side of the index finger (Fig. 2A). Dominant SPB also sent important branches to the thumb, in 10 out of 11 cases, and in regard to their presence two subtypes could be distinguished.

Type A1. With only ulnaris pollicis palmar artery (UPPA) existing, subtype found in $6(17.1 \%)$ hands, destined for the medial palmar side of the thumb. Its average diameter was 1.15 (range 1.0-1.3) mm (Fig. 2B, Table 1).
Type A2. With both of them, ulnaris and radialis pollicis palmar arteries (UPPA and RPPA) departing either individually or by a common stem, supplying both the medial and lateral, palmar sides of the thumb, in $4(11.4 \%)$ cases. Their respective average diameters were 1.3 (range 1.0-1.4) $\mathrm{mm}$ for the ulnaris and 1.2 (range 1.0-1.35) $\mathrm{mm}$ for the radialis pollicis branch of SPB (Figs. 1, 2C, Table 1).

Type B. The hypoplastic SPB, present in most cases 24 (68.6\%), was defined as being below $1.7 \mathrm{~mm}$ in diameter, ranging from 0.8 to 1.5 (mean 1.17) $\mathrm{mm}$ (Table 1). It gave off long and delicate cutaneous branches for the vascularisation of the skin of the thenar eminence, as well as for muscles of the same region, and completely exhausted within the thenar area (Fig. 3A).

Our original classification of the SPB into two groups was based on a detailed study of 35 corrosion casts and 20 formol-injected dissected hands. Starting from the smallest diameter of $0.8 \mathrm{~mm}$ up to the $1.5 \mathrm{~mm}$, the observed SPBs were vessels supplying the muscles and skin of the thenar area. The arteries in our collection, which had the calibre of $1.7 \mathrm{~mm}$ and above, always irrigated a much larger field, including the index finger and the thumb. We concluded that a larger SPB, starting from the observed $1.7 \mathrm{~mm}$, are well developed and potentially more important for the hand supply. The observed rule is that only all well-developed SPBs with diameters above $1.7 \mathrm{~mm}$ 
were directly, side to side, connected to the SPA by a smaller in diameter communicating branch (CB) (Figs. 1, 2).

Anastomoses were present and numerous in the irrigation territory of the SPB supply, mostly in the thenar region. Communicating branch with SPA of the UA, as a single vessel, was reserved only for cases with a large and well-developed SPB (31.4\%). The cases with a thinner and hypoplastic SPB were more numerous (68.6\%). All those examined hands contained in the subcutaneous tissue well ramified, slender cutaneous branches showing two groups of anastomotic connections with surrounding vessels. Lateral group of parallel anastomoses communicated with branches of thenar arteries deriving from the anatomical snuff-box portion of the RA. Distal group of anastomotic channels showed multiple small arteries making contacts with the SPA of the UA. From the thenar vascular network short twigs pierced the thenar fascia, rapidly dividing in all directions in a stellate pattern, finally reaching the skin (Figs. 3A, B).

\section{DISCUSSION}

Despite a large number of articles presenting anatomical characteristics of superficial and deep palmar arches, some palmar arteries were only partially described, for example, the thenar arteries coming from the anatomical snuff-box portion of RA. This is obviously true for the SPB of the RA, which was in previous reports only mentioned as a part of SPA $[2,5,13,14,23,26]$.

Our original contribution to the morphometric and topographic classification of the SPB into two groups was a result of a more detailed analysis of the SPB itself, then in a previous observation of the thenar eminence blood supply [26]. Measuring the calibres and comparing the frequency of well-developed SPBs in our present series of 35 corrosion casts, which was $31.4 \%$, with the earlier incidence of $40 \%$ of prominent SPB in 30 human hands, no statistically significant difference was found ( $\chi^{2}$ with Yates correction 0.212 , two-tailed $p=0.6453)$, confirming and supporting our methodological approach. In this present analysis we also measured the diameters of the branches: RIA, UPPA and RPPA, which were in average above $1 \mathrm{~mm}$ (Table 1), showing numerically that full size vessels arising from the well-developed SPB are more important in supplying the index and thumb fingers in comparison to a small communicating connection with the SPA of the ulnar artery.

Our data obtained in this study showed that the SPB is usually a subcutaneous vessel (in 75\%), and in most cases, $(68.6 \%)$, with the diameter below $1.7 \mathrm{~mm}$, with the average value of $1.17 \mathrm{~mm}$. Although being small in absolute terms, this is the largest artery of the thenar eminence with a local field of supply, the skin and muscles. Hands with this small, hypoplastic SPB did not show the typical CB for the SPA. Instead of a $C B$ we identified slender anastomotic vessels, lateral, and distal group for the connection with SPA. Analysing our description of the most common appearance of the SPB of the RA with the other reports, we were able to find only one similar illustration. Omokawa et al. [17] found a typical distribution of the SPB branches, supplying only the radial aspect of the thenar eminence, in $83.3 \%$ of specimens, the incidence higher than one in the current study (68.6\%).

The incidence of SPB with the diameter superior to 2.00 (mean 2.4 ) $\mathrm{mm}$ in $16.7 \%$ of hands was reported by them, or, according to Bilge et al. [2], in 14\%. In those cases both groups stated the dominant supply to the thumb and index finger including the whole area of the thenar eminence, with the incidence lower than in our results $(31.4 \%)[2,17]$. However, they did not distinguish subtypes with different branching patterns, within the well-developed SPB type, as we did. Our established value of $1.7 \mathrm{~mm}$ for the SPB calibre, was a point of depart from which a typical, hypoplastic SPB became larger, dominant vessel, with larger field of supply covering index finger obligatory, and parts of the thumb ( $A 1$ and $A 2$ subtypes). Further, those two studies did not note the rule that $C B$ existed only in cases with dominant SPB.

We agree and accept descriptions of the positions and counting of three or four common palmar digital arteries arising from the SPA, according to previous authors and Singh et al. [23], and from all other articles and textbooks (if explained). Generally speaking, the first common palmar digital artery runs between the index and middle finger. On the other hand, if the SPA is longer and its terminal part finally bifurcates into the RIA and princeps pollicis artery (PPA), this common part is considered as the first common palmar digital artery [5]. In most cases the PPA is a branch of the RA [15]. Our findings showed that the well-developed SPBs provided origin for the palmar thumb arteries, usually branches of PPA, together with the RIA. The small contribution of a CB with the UA is visible on corrosion cast specimens; in other words, a potential first common palmar digital artery is not really a branch of SPA, rather a full continuation of the well-developed SPB. 
The mean diameter of the RIA was $1.54 \pm 0.4 \mathrm{~mm}$, as reported by Bilge et al. [2]. In the current study, the calibre of the RIA was on the average 1.2 (range 1.0-1.5) $\mathrm{mm}$.

According to the findings of Miletin et al. [15], SPB can be a dominant source of the thumb blood supply in $7.69 \%$ of the cases. This is in accordance with our $11.4 \%$ cases in which the SPB branches supplied both, medial and lateral palmar sides of the thumb.

Existing differences between the data from the literature and our findings might be due to different methodology applied in the studies. Mentioned authors injected hands and dissected them, in contrast to our use of corrosion casts of the arterial system of the hands. Corrosion casts of the whole hands are very accurate specimens, representing very realistically the spatial anatomical arrangement of injected arteries, more precisely than any dissection. Direct visualisation and measurements of the luminal diameter of even tiny branches is possible [9].

Our findings regarding the average calibre of the SPB of 1.5 (range $0.8-2.7$ ) $\mathrm{mm}$, are very similar to the ones reported by Omokawa et al. [17] (mean 1.4, range $0.8-3 \mathrm{~mm})$, Bilge et al. [2] (1.67 mm), and Fazan et al. [5] (1.55 mm).

Instead of studies of the anatomical properties of the SPB, the scientific articles were focused on the superficial vascular pattern of the hand, and traditionally defined one of the complete arches types of SPA as cases when it was in connection to the SPB of RA $[2,5,6,13,23]$. Habitually, the complete SPA was found in: $40 \%$ of cases according to Loukas et al. [13], 34\% Bilge et al. [2], 48\% Fazan et al. [5], 35.5\% Gellman et al. [9], 27.4\% Feigl et al. [6], 44\% Singh et al. [23], and 53.3\% according to Omokawa et al. [17]. In general, our results regarding the existence of $C B$, and the so-called traditional or complete SPA, in $31.4 \%$ of cases are in accordance with the findings of the afore mentioned authors.

The SPB of the RA is a very constant artery, present in $100 \%$ of cases, despite the widely described variations in size, area of supply and connections with the SPA. Klimek-Piotrowska et al. [11] described unusual variations of the arteries of two upper limbs on the same cadaver. In both hands the SPB were missing. Such anatomical variations and of our findings related to the distribution area of the dominant SPB (in contrast to the small hypoplastic type), could be explained on the basis of their embryological development. This theory suggests that vascular varia- tions in the upper limb are formed by modifying the primary capillary plexus by means of progression or regression within the framework of haemodynamic predominance of feeding arteries of the hand: radial, ulnar, median and common interosseous [11, 19].

Our study presents the SPB as a constant and well-ramified artery, branching under the skin of the thenar eminence. The thenar flap is a popular surgical technique in the reconstruction of fingertip injuries with loss of soft tissue and exposed bone [24]. In cases of patients who have had two fingertip amputations, one can apply the double thenar flap technique, as reported in the literature [12]. Our results confirmed that the SPB could be used for the safe elevation of a fasciocutaneous flap [17]. The always present distal anastomoses in cases of small size SPB, and larger arterial connections in cases with the dominant SPB, theoretically allow for the flap to be transferred as a reverse-pedicled, in order to cover the larger defects of the palm, index finger, and thumb $[10,22]$. Use of a free microvascular thenar flap for reconstruction of intraoral soft tissues has also been reported [8].

Harvest of the RA and use of its free grafts for a coronary artery bypass could potentially induce arterial insufficiency because of inadequate collateral circulation. The Doppler studies obtained the RA and UA diameters and flows, both preoperative and postoperatively [3]. They demonstrated good hand perfusion and an increase in UA flow velocity and diameter based on the functional compensatory changes and anastomotic vascular network. We proved the existence of multiple anastomotic vessels between the SPB and SPA, as we already stated. Therefore, including the substantial and complete deep palmar arch of the $\mathrm{RA}$, the potential for adequate collateral circulation is warranted. The limitation of our study could be the sample size, i.e. the number of the available hand specimens, in order to establish precise definitions on the variations of these structures.

\section{CONCLUSIONS}

We have presented detailed morphometric material describing the SPB of RA, and explained the differences between the hypoplastic and the well-developed type of artery. Based on the examined material we concluded that a SPB with diameter inferior to $1.7 \mathrm{~mm}$ was locally arborising within the thenar area, in contrast to the SPBs with a calibre of $1.7 \mathrm{~mm}$ and larger, the latter extending into the index finger and thumb areas. Furthermore, we discussed in detail 
the cutaneous branches of this artery, their area of supply and their anastomotic vessels. It is our hope that such useful anatomical descriptions and the obtained data might have implications in orthopaedic, microvascular, plastic and reconstructive, and cardiothoracic surgery.

\section{Acknowledgements}

This work was supported by grant no. 175030 from the Ministry of Education, Science and Technological Development of the Republic of Serbia.

\section{REFERENCES}

1. Baetz L, Satiani B. Palmar arch identification during evaluation for radial artery harvest. Vasc Endovascular Surg. 2011; 45(3): 255-257, doi: 10.1177/1538574411399159, indexed in Pubmed: 21478246.

2. Bilge O, Pinar Y, Ozer MA, et al. A morphometric study on the superficial palmar arch of the hand. Surg Radiol Anat. 2006; 28(4): 343-350, doi: 10.1007/s00276-006-0109-9, indexed in Pubmed: 16642281.

3. Brodman RF, Hirsh LE, Frame R. Effect of radial artery harvest on collateral forearm blood flow and digital perfusion. J Thorac Cardiovasc Surg. 2002; 123(3): 512-516, doi: 10.1067/mtc.2002.119885, indexed in Pubmed: 11882824.

4. Cormack GC, Lamberty BGH. The arterial anatomy of skin flaps. Churchill Livingstone. 1994: 210-213.

5. Fazan VP, Borges CT, Da Silva JH, et al. Superficial palmar arch: an arterial diameter study. J Anat. 2004; 204(4): 307-311, doi: 10.1111/j.0021-8782.2004.00281.x, indexed in Pubmed: 15061757.

6. Feigl GC, Petrac M, Pixner T, et al. The superficial palmar arch and median artery as an example of misleading results due to a small number of investigated specimens or the use of different classifications. Ann Anat. 2012; 194(4): 389-395, doi: 10.1016/j.aanat.2011.10.013, indexed in Pubmed: 22196998.

7. Fitoussi $F$, Ghorbani $A$, Jehanno $P$, et al. Thenar flap for severe finger tip injuries in children. J Hand Surg Br. 2004; 29(2): 108-112, doi: 10.1016/j.jhsb.2003.10.006, indexed in Pubmed: 15010153.

8. Gaggl A, Bürger $H$, Brandtner $C$, et al. The microvascular thenar flap as a new possibility for super-thin soft tissue reconstruction in the oral cavity--initial clinical results. $\mathrm{Br}$ J Oral Maxillofac Surg. 2012; 50(8): 721-725, doi: 10.1016/j. bjoms.2012.02.001, indexed in Pubmed: 22365744.

9. Gellman H, Botte MJ, Shankwiler J, et al. Arterial patterns of the deep and superficial palmar arches. Clin Orthop Relat Res. 2001(383): 41-46, indexed in Pubmed: 11210968.

10. Hugon S, Castus $P$, Schoofs M. Index reconstruction by means of a fasciocutaneous thenar flap. Plast Reconstr Surg. 2010; 126(1): 43e-44e, doi: 10.1097/PRS.0b013e3181dab473, indexed in Pubmed: 20595849.

11. Klimek-Piotrowska W, Pacholczak R, Walocha J. Multiple variations of the arterial pattern in upper extremities: a case report and embryological pathogenesis. Clin Anat.
2013; 26(8): 1031-1035, doi: 10.1002/ca.22171, indexed in Pubmed: 23037918.

12. Kwon YJ, Ahn BM, Lee JS, et al. Reconstruction of two fingertip amputations using a double thenar flap and comparison of outcomes of surgery using a single thenar flap. Injury. 2017; 48(2): 481-485, doi: 10.1016/j.injury.2016.12.023, indexed in Pubmed: 28057323.

13. Loukas M, Holdman D, Holdman S. Anatomical variations of the superficial and deep palmar arches. Folia Morphol. 2005; 64(2): 78-83, indexed in Pubmed: 16121323.

14. McLean KM, Sacks JM, Kuo YR, et al. Anatomical landmarks to the superficial and deep palmar arches. Plast Reconstr Surg. 2008; 121(1): 181-185, doi: 10.1097/01. prs.0000293863.45614.f9, indexed in Pubmed: 18176219.

15. Miletin J, Sukop A, Baca V, et al. Arterial supply of the thumb: Systemic review. Clin Anat. 2017; 30(7): 963-973, doi: 10.1002/ca.22973, indexed in Pubmed: 28791730.

16. Moore KL, Dalley AF, Agur AMR. Clinicaly oriented anatomy. Lippincott Williams and Wilkins, Baltimore. 2014: 759-782.

17. Omokawa S, Ryu J, Tang JB, et al. Vascular and neural anatomy of the thenar area of the hand: its surgical applications. Plast Reconstr Surg. 1997; 99(1): 116-121, indexed in Pubmed: 8982194.

18. Pistre V, Pelissier P, Martin D, et al. Vascular blood supply of the dorsal side of the thumb, first web and index finger: anatomical study. J Hand Surg Br. 2001; 26(2): 98-104, doi: 10.1054/jhsb.2000.0538, indexed in Pubmed: 11281658 .

19. Rodríguez-Niedenführ M, Burton GJ, Deu J, et al. Development of the arterial pattern in the upper limb of staged human embryos: normal development and anatomic variations. J Anat. 2001; 199(Pt 4): 407-417, indexed in Pubmed: 11693301.

20. Ross AC. Wrist and Hand. In: Standring S (Editor-in-Chief), Gray's anatomy. The anatomical basis of clinical practice. Elsevier Limited, Philadelphia. 2016: 862-897.

21. Salmon M. Arteries of the skin. Churchill Livingston, London-New York. 1988: 32-34.

22. Seyhan T. Reverse thenar perforator flap for volar hand reconstruction. J Plast Reconstr Aesthet Surg. 2009; 62(10): 1309-1316, doi: 10.1016/j.bjps.2008.03.061, indexed in Pubmed: 18667372.

23. Singh S, Lazarus L, De Gama BZ, et al. An anatomical investigation of the superficial and deep palmar arches. Folia Morphol. 2017; 76(2): 219-225, doi: 10.5603/ FM.a2016.0050, indexed in Pubmed: 27665957.

24. Thanik V, Shah A, Chiu D. A technique for tripartite reconstruction of fingertip injuries using the thenar flap with bone and nail bed grafts. J Hand Surg Am. 2017; 42(12): 1040.e1-1040.e7, doi: 10.1016/j.jhsa.2017.09.011, indexed in Pubmed: 29198319.

25. Vidić B, Milisavljević M, Maliković A. Atlas of the human body, central nervous system and vascularization. Elsevier, Academic Press, London. 2017: 14-24.

26. Zekavica A, Milisavljević M, Erić $D$, et al. Vascular anatomy of the thenar eminence: its relevance to a pedicled or free thenar flap. Folia Morphol. 2017; 76(2): 232-238, doi: 10.5603/FM.a2016.0077, indexed in Pubmed: 28026846. 